\title{
reviscafuences
}

ISSN: 1575-7072 | e-ISSN: 2172-7775

Páginas: 238-250

Recibido: 2019-04-30

Revisado: 2019-11-24

Aceptado: 2020-01-14

Preprint: 2020-07-15

Publicación Final: 2020-09-15 www.revistascientificas.us.es/index.php/fuentes/index

DOI: https://doi.org/10.12795/revistafuentes.2020.v22.i2.01

\section{Calidad de los aprendizajes de estudiantes de pedagogía: influencia del modelo educativo}

\author{
Quality of learning of students of pedagogy: influence of the educational model
}

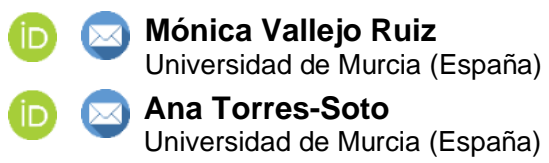

\section{Resumen}

Son numerosos los trabajos que han señalado como factor distintivo de calidad en educación los aprendizajes de los estudiantes. Este estudio analiza el aprendizaje de estudiantes de Pedagogía de la Región de Murcia al finalizar sus estudios universitarios, con objeto de valorar la calidad de los aprendizajes desarrollados con la incorporación al Espacio Europeo de Educación Superior. Se trata de un estudio de caso en el que han participado 47 estudiantes, a través de cuestionarios abiertos creados ad hoc. Los resultados denotan, claramente, dos perfiles diferenciados de alumnos en función de los aprendizajes desarrollados (aprendizaje superficial y aprendizaje profundo). El perfil que mayor número de estudiantes agrupa muestra un aprendizaje superficial (comprensión baja y resolución simple de problemas). Se comparte con estudios recientes la necesidad de que el profesorado favorezca en los estudiantes estrategias y enfoques de aprendizaje que lleven a la comprensión y a la significación personal, así como favorecer el desarrollo de estrategias efectivas de enseñanza-aprendizaje fundamentadas en las buenas prácticas.

\section{Abstract}

Numerous studies have pointed to student learning as a distinctive factor of quality in education. This study analyses the learning of Pedagogy students from the Region of Murcia at the end of their university studies, with the aim of assessing the quality of the same with the incorporation into the European Higher Education Area. This is a case study in which 47 students participated, through open questionnaires created ad hoc. The results show two differentiated profiles of students according to the learning developed (superficial learning and deep learning). Superficial learning clusters a greater number of students (low comprehension and simple problem solving). Recent studies share the need for teachers to favour learning strategies and approaches in students that lead to understanding and personal significance, as well as to favour the development of effective teaching-learning strategies based on good practices.

\section{Palabras clave / Keywords}

aprendizaje; calidad de la educación; educación superior; enseñanza; evaluación del aprendizaje. learning; quality of education; higher education; teaching; learning assessment. 


\section{Introducción}

En un contexto en el que la educación se considera como una de las principales herramientas de desarrollo humano, económico y social (Delors, 1996; Gabay, 2015), la práctica educativa y el aprendizaje de los estudiantes debieran significar autonomía personal y profesional, resiliencia, empoderamiento y autosuficiencia, y aspiraciones adecuadas que reviertan en mejores resultados académicos. Unido a ello y, sobre todo en la etapa universitaria, adquiere valor imprescindible el desarrollo de aprendizajes profundos y de calidad para un desarrollo profesional adecuado.

De igual modo, son varios los trabajos que han señalado como indicador distintivo de calidad de la educación los aprendizajes de los estudiantes (Duque, Celis, Díaz y Gómez, 2014; Guzmán, 2011; Roys y Pérez, 2018; Stewart, 2012). Sobre este último aspecto, algunas investigaciones realizadas en el ámbito internacional (McDermott, 1984; West, 1988; Ramsden, 2003; Grácio, Chaleta y Rosário, 2005) sobre la calidad de los aprendizajes de los estudiantes universitarios han manifiestado un cuadro desolador de la Educación Superior. Estas investigaciones revelan que los estudiantes son capaces de describir amplias cantidades de información y conceptos relacionados con las disciplinas objeto de estudio pero muestran ciertas dificultades para demostrar la comprensión de esos contenidos y para aplicarlos a diversas situaciones de la vida cotidiana y propias de la profesión.

Esta realidad, junto con un modelo educativo excesivamente academicista y arraigado característico del sistema educativo superior, ponen en el punto de mira un problema multifacético que se aleja notoriamente de las pretensiones referidas a la calidad de los aprendizajes del Espacio Europeo de Educación Superior (en adelante, EEES). Se aleja también de planteamientos competenciales como la creatividad, la resolución de problemas, el liderazgo o el razonamiento, relacionadas con la utilidad del conocimiento más allá de lo estrictamente académico (Martínez y Carmona, 2009), y del valor social de la educación, entendido este valor como las motivaciones, expectativas, valores y significación que genera el aprendizaje en sí mismo al alumnado.

Considerando estos planteamientos, el estudio que se presenta valora la formación de los estudiantes de Pedagogía de la Universidad de Murcia (España), ahondando en su capacidad para hacer frente a diferentes situaciones profesionales y continuos cambios que puedan acontecer en su quehacer profesional, teniendo en consideración que la mayoría de estos estudiantes habían participado en diversos cursos piloto para la adaptación al modelo formativo del EEES. Como es sabido, desde que se iniciara el proceso de convergencia europea se viene requiriendo que las Universidades formen a sus estudiantes en la construcción de aprendizajes comprensivos, significativos, discutibles, fundamentados, científicos y explícitos (Pérez-Gómez, 2009; Olmedo, 2013) que les permitan hacer frente a imprevisibles cambios futuros y a las necesidades que los avances de la sociedad del conocimiento plantean (Arias y Fidalgo, 2013; Riesco, 2008). Este artículo profundiza en la evaluación de dichos aprendizajes y discute, finalmente, la importancia de desarrollar estrategias efectivas para mejorar la capacidad de aprender de los estudiantes y la capacidad de enseñar de los docentes.

\subsection{Importancia del modelo educativo y la orientación hacia el aprendizaje de los estudiantes}

En la actualidad, los docentes tienden a enfrentarse a una emergente concepción constructivista del aprendizaje caracterizada por el reto de replantear sus prácticas de enseñanza e introducir metodologías activas y el desarrollo de estrategias cognitivas complejas en sus estudiantes (Vázquez-García, 2015). Las directrices en las que se sustenta el EEES reclaman la necesidad de renovar curricular y metodológicamente las prácticas docentes en el contexto universitario como vías para mejorar la calidad de la enseñanza (GilGalván, 2018). Así, el papel fundamental de los docentes es el de mediar y articular buenos entornos y experiencias de aprendizaje (Forés y Subias, 2017; Monereo y Pozo, 2003) en los que los estudiantes aprendan de manera significativa y profunda. Se acentúa, en consecuencia, una mirada crítica hacia la práctica docente tradicional, centrada en el aprendizaje memorístico y la transmisión superficial de los contenidos (Biggs y Tang, 2011; Pozo y Monereo, 2009; Zabalza, 2011), que poco o nada se encaminan a lograr un aprendizaje significativo y competencial en los estudiantes.

En las últimas décadas se han venido desarrollando diversas investigaciones, denominadas SAL (Students Aproaches to Learning), que han relacionado la calidad de los aprendizajes que construyen los estudiantes con los enfoques y estrategias que ponen en marcha para aprender (Biggs y Tang, 2011; Buendía y Olmedo, 2003; Entwistle, 1986; Esquivel, Rodríguez y Padilla, 2009; Gadelrab, 2011; Gargallo, Garfella, Sahuquillo, Verde et al., 2015; Maquilón y Hernández-Pina, 2011; Marton y Saljo, 1976ab; Rosário y Almeida, 2005; Roys 
y Pérez, 2018; Salim, 2006). Los enfoques de aprendizaje reflejan las intenciones del estudiante ante el aprendizaje y la manera de enfrentarse a él. Conceptualmente, se define el enfoque superficial como aquel que utilizan los alumnos que tienen la intención de liberarse de la tarea con el mínimo esfuerzo, aunque dando la sensación de satisfacer los requisitos. Estos estudiantes se limitan a memorizar y, como consecuencia, tienen una concepción reproductiva del aprendizaje. Así, construyen un aprendizaje de bajo nivel cognitivo, acompañado de una escasa comprensión y una mínima actitud reflexiva. El enfoque profundo, por el contrario, es definido como aquel que utilizan los alumnos que abordan las tareas de aprendizaje de forma adecuada y significativa, mediante el uso de estrategias y actividades cognitivas complejas. Estos estudiantes muestran un mayor interés por comprender el significado de las tareas, utilizan la comprensión y la lógica y la crítica en sus argumentaciones. Existe un tercer enfoque, denominado estratégico, que se describe como aquel mediante el cual los estudiantes se limitan a conseguir buenas calificaciones, centrándose en descubrir lo que el profesor demanda y en cómo obtener la mejor calificación posible. Estos enfoques de aprendizaje se encuentran directamente relacionados con la calidad del aprendizaje.

Ritchhart, Church y Morrison (2014) también describen la relación existente de dichos enfoques y la calidad del aprendizaje construido. Así, la adopción de un enfoque superficial converge en aprendizajes memorísticos y la adopción de un enfoque profundo, en un aprendizaje significativo y profundo. Este planteamiento sitúa al conocimiento en un continuo que se desarrolla entre dos polos, desde la superficialidad hasta la profundidad, al igual que ocurre con los enfoques de aprendizaje.

Gargallo, Suárez, García, Pérez, et al. (2012) señalan que la adopción de un enfoque u otro viene determinado, a su vez, por las estrategias y metodologías adoptadas por los docentes en sus prácticas educativas. De esta manera, los estudiantes hacen uso de enfoques profundos de aprendizaje cuando los planteamientos metodológicos adoptados por el profesorado se centran en el aprendizaje y se alinean metodologías y evaluación adecuadas. Por el contrario, cuando los profesores utilizan únicamente metodologías expositivas y examen final como método de evaluación, los estudiantes se decantan por enfoques superficiales hacia el aprendizaje.

En este sentido, se considera que tanto las aspiraciones, metas y motivaciones que los discentes poseen, como las estrategias metodológicas desarrolladas por el profesorado, afectarán directamente al proceso de enseñanza y aprendizaje y a sus resultados académicos (Sancho-Álvarez, Jornet y Perales, 2014; SanchoÁlvarez, Jornet y González-Such, 2016). Esto es, como se ha mencionado, estudiantes que pongan en marcha enfoques de aprendizaje profundos, que muestren interés y motivación por aprender, que tengan ciertas aspiraciones personales y profesionales, están asociados a aprendizajes de mayor calidad. Y si además los itinerarios de aprendizaje han sido bien planteados por los docentes, les llevarán al desarrollo de competencias emprendedoras. Estas competencias se caracterizan, de acuerdo con Martínez y Carmona (2009, p.95), por "la autonomía de la persona y se orientan hacia la autorrealización del sujeto, como vía para la realización de un proyecto vital centrado en la producción de bienes y servicios que satisfagan las necesidades de la comunidad". Todo ello requiere que las propuestas didácticas actuales insistan en orientaciones profundas del aprendizaje. Pero, ¿realmente es así? ¿Se están desarrollando aprendizajes profundos, significativos y relevantes en los estudiantes universitarios?

A pesar de todo, si bien la relación entre el uso de modelos educativos centrados en el aprendizaje del estudiante y los enfoques de aprendizaje adoptados han sido investigados y evidenciados por numerosas investigaciones, la relación directa entre el uso de modelos educativos centrados en el aprendizaje del estudiante y el propio aprendizaje que se deriva de esa enseñanza ha tenido una constatación más débil (González y Cutanda, 2017; Hightower, Delgado, Lloyd, Wittenstein, et al., 2011). En este estudio se trata de conocer el aprendizaje del alumnado de Pedagogía y su posible vinculación con el uso del modelo educativo utilizado. Así pues, los objetivos que se persiguen en este trabajo son, en primer lugar, valorar el grado de profundización y comprensión del conocimiento construido por los estudiantes a partir de la taxonomía SOLO (Structure of the Observed Learning Outcome) de John Biggs; en segundo lugar, precisar perfiles de alumnos atendiendo al conocimiento analizado; $y$, en tercer lugar, conocer la existencia o no de diferencias significativas según género y entre estudiantes que han participado cursos piloto de adaptación al EEES y los que no lo han hecho.

\section{Método}

Se ha optado por un diseño eminentemente fenomenológico, propicio para reconocer significados en los discursos de los participantes mediante el análisis de temas específicos (Bisquerra, 2012). Específicamente, la estrategia utilizada en el diseño de la investigación ha sido el estudio de caso, teniendo éste una finalidad 
evaluativa (Pérez-Serrano, 1994). Precisamente, se ha pretendido la búsqueda de información relevante por parte de los participantes en un escenario "natural", para no alterar el sentido o significado que allí confieren a sus experiencias de aprendizaje (Merriam, 2016). Se considera esta metodología como particularmente relevante en la realización de este estudio de caso, dado que permite crear conjeturas y proposiciones que podrían ser abordadas en estudios posteriores con un mayor alcance, profundidad y detalle (George y Bennett, 2005).

Se han complementado los análisis cualitativos realizados con algunos procedimientos y técnicas de análisis propias de los enfoques cuantitativos, con el objetivo de agrupar y reconocer patrones en los estudiantes en función de los niveles de aprendizaje evidenciados.

\subsection{Participantes}

La muestra participante en la investigación, obtenida a partir de un proceso de muestreo no probabilístico intencional, la conforman un total de 47 estudiantes del título de Pedagogía, de los cuales 43 son mujeres y 4 son hombres. Estos estudiantes reúnen una característica singular: comenzar la titulación de Pedagogía en el mismo curso académico y estar matriculado en el último curso en el momento de la recogida de la información. De todos ellos, 42 estudiantes participaron en varios cursos piloto a lo largo de su formación y 5 de ellos no habían sido partícipes, en ningún curso académico, de dichos cursos.

Esta investigación recoge la población completa de estudiantes de Pedagogía de la Región de Murcia de un curso académico concreto. Pero al ser una muestra pequeña de participantes, no está exento de limitaciones. Si bien se considera que los hallazgos pueden tener implicaciones para el desarrollo de propuestas de formación ajustadas a las necesidades formativas del profesional de Pedagogía y podrían servir, asimismo, para comprender otros casos o situaciones con características similares.

\subsection{Instrumentos de recogida de información}

Se ha elaborado un instrumento de recogida de información, creado ad hoc, para dar respuesta a la complejidad y los requerimientos del contexto de estudio. Concretamente, se han diseñado dos instrumentos: un cuestionario abierto y otro semiestructurado. El motivo de su elección como instrumento de recogida de información responde a las siguientes razones: por un lado, se considera que, al tratarse de un documento escrito, favorece la expresión de los estudiantes -al ser una de las modalidades de expresión más frecuentes en el ámbito académico-; por otro lado, se han tenido en cuenta las posibilidades que presentan estas técnicas de recogida de información para acceder a una gran cantidad de personas a la vez y en un periodo relativamente corto de tiempo (García-Córdoba, 2004), más cuando se pretende salvar la posibilidad de comentarios entre la muestra participante sobre la temática de las preguntas planteadas.

Para el diseño de los instrumentos se han tenido en cuenta dos dimensiones que se pretendían estudiar: por un lado, el conocimiento declarativo del alumnado y, por otro lado, el conocimiento funcional (véase Biggs, 2005). A este respecto, se ha configurado un patrón de cuestiones (Tabla 1), asegurando en su presentación a los estudiantes seguir el orden y continuidad de dichas dimensiones. Tal y como figura en la Tabla 1, el Instrumento 1 recoge diez cuestiones referidas a diez temas que habían sido relevantes en la formación de los estudiantes1. Por su parte, el Instrumento 2 reune tres casos prácticos sobre distintas temáticas estudiadas en diversas asignaturas de la Titulación.

\footnotetext{
${ }_{1}$ Con anterioridad a la elaboración y administración de los Instrumentos 1 y 2, los estudiantes cumplimentaron un cuestionario abierto en el cual debían plasmar los contenidos que habían aprendido en cada una de las materias que habían cursado. Del análisis de la información recabada, se utilizaron los contenidos más frecuentemente recordados por los estudiantes para diseñar los instrumentos que en este estudio presentamos.
} 


\section{Tabla 1}

Instrumentos de recogida de información

\begin{tabular}{ll} 
Instrumentos & Patrón de cuestiones planteadas \\
\hline Instrumento 1 & 1. Identifica y selecciona un autor (pedagogo, filósofo, psicólogo, etc.) que consideres importante \\
en tu formación como pedagogo y explica cuáles han sido sus aportaciones más relevantes. \\
2. ¿Qué entiendes por currículum? \\
3. ¿Qué leyes educativas crees que han tenido más impacto en el sistema educativo español? \\
¿Por qué? \\
4. ¿Qué programas informáticos de análisis de datos conoces? ¿Cuáles son sus ventajas e \\
inconvenientes? \\
5. Explica qué características posee un niño con Síndrome de Down y qué implicaciones \\
educativas tendría su escolarización. \\
6. Explica qué entiendes por modelo de orientación, selecciona uno de los modelos existentes y \\
sus características básicas. \\
7. ¿Qué es un proyecto de intervención socioeducativa? \\
8. Expresa qué entiendes por aprendizaje y qué tipo de aprendizajes conoces. \\
9. ¿Podrías identificar un aspecto (organización, estructura, etc.) de otro sistema educativo \\
europeo que creas puede ser interesante adoptarlo en el sistema educativo español? \\
10. Define educación en valores. \\
1. Detección de un alumno con altas capacidades \\
2. Importancia e implicaciones de la evaluación en educación. \\
3. Formación continua del profesorado
\end{tabular}

Concretamente, los tres casos planteados en el Instrumento 2 han sido diseñados de manera que, a partir de las respuestas de los estudiantes, se pueda valorar su capacidad para el desarrollo de ciertas funciones generales de los pedagogos. De acuerdo con el Libro Blanco (2005) se establecen tres funciones generales propias del pedagogo: analizar aspectos que conforman situaciones educativas en diferentes contextos formativos; diseñar programas, acciones y proyectos adaptados a los contextos analizados; realizar un seguimiento y evaluación a los programas, acciones y proyectos diseñados e implementados para cada contexto educativo. En consideración con estas funciones se ha planteado un primer caso para recoger información acerca de la capacidad del alumnado para analizar, determinar y diagnosticar a un alumno con altas habilidades, así como para plantear medidas para su respuesta educativa. Un segundo caso práctico en que el alumnado ponga de manifiesto su capacidad de asesoramiento y orientación a profesores sobre los tipos distintos tipos de evaluación, los instrumentos de evaluación y las implicaciones de su uso en la función docente. $Y$ un tercer caso práctico en el que se plantean algunas cuestiones que pretenden conocer la capacidad del alumnado para analizar diversas situaciones, así como para plantear acciones y medidas para la formación continua de profesores.

Ambos instrumentos han sido validados a través de un procedimiento inter-jueces y a partir de la validez de uso (Navarro, 2012). En relación a la fiabilidad, el Coeficiente Alfa de Cronbach arrojó un coeficiente de 0.814, lo cual indica, de acuerdo con Bisquerra (2012), una fiabilidad alta.

\subsection{Construcción del sistema de categorías}

Con el fin de dar respuesta a los objetivos de investigación planteados se ha elaborado, previamente al análisis de los datos, un sistema de categorías para afrontar el análisis de las respuestas de los estudiantes a partir de su complejidad estructural, atendiendo a cinco niveles de progresión (1 menor y 5 mayor) y trece categorías de análisis (referidas a las diez cuestiones planteadas en el Instrumento 1 y los tres casos prácticos planteados en el Instrumento 2).

El análisis de la información de cada una de las trece categorías se fundamenta en las aportaciones de Biggs y Collis (1982), Biggs (2005) y Biggs y Tang (2011). Inicialmente, Biggs y Collis propusieron la taxonomía SOLO en la que identificaron cinco niveles de complejidad estructural para valorar la calidad de los aprendizajes de los estudiantes (preestructural (1), uniestructural (2), multiestructural (3), realacional (4) y de abstracción extendida (5)). De acuerdo con los autores, las respuestas de los estudiantes pueden situarse en 
un continuo que iría desde respuestas simples e irrelevantes sin estructuración ni coherencia (nivel 1) hasta respuestas que utilizan la reconceptualización, la reflexión e incluso la inferencia del conocimiento a diversas situaciones y contextos (nivel 5). Para adaptar cada uno de los niveles a las categorías de análisis, se ha redefinido cada uno de los niveles adaptándolos a la temática de cada una de las categorías de análisis. En la siguiente tabla figura un ejemplo de redefinición de los niveles de acuerdo con una categoría a analizar (Tabla 2).

Tabla 2

Matriz para el análisis de las respuestas de los alumnos (Ejemplo de categoría 5)

\begin{tabular}{|c|c|}
\hline Categoría & Categoría 5. Características del Síndrome de Down e implicaciones educativas \\
\hline Nivel 1 & $\begin{array}{l}\text { No identifica características propias de las personas con Síndrome de Down y no enuncia las } \\
\text { implicaciones educativas que podría acarrear su escolarización. (C5-N1) }\end{array}$ \\
\hline Nivel 2 & $\begin{array}{l}\text { Es capaz de identificar algunas características, aunque de forma muy limitada. Los contenidos que } \\
\text { maneja son cotidianos, no utiliza conceptos elaborados. No es capaz de analizar las implicaciones } \\
\text { educativas que tendría su escolarización, y si lo hace, de manera muy descriptiva y poco coherente. } \\
\text { (C5-N2) }\end{array}$ \\
\hline Nivel 3 & $\begin{array}{l}\text { Enuncia las características que identifican a un niño con Síndrome de Down diferenciando entre físicas } \\
\text { y psíquicas. Es capaz de enunciar, de manera muy generalista, algunas de las implicaciones más } \\
\text { importantes en la escolarización de estos niños aunque sin contextualizar la información y sin ofrecer } \\
\text { argumentos razonados ni conclusiones. Emplea un estilo meramente descriptivo. (C5-N3) }\end{array}$ \\
\hline Nivel 4 & $\begin{array}{l}\text { Es capaz de relacionar las características de estos niños con las implicaciones educativas que se } \\
\text { derivan de su escolarización. Ofrece algunas explicaciones teóricas para justificar sus argumentos. } \\
\text { Llega a ofrecer conclusiones y algunos juicios personales -aunque no muy elaborados- sobre la } \\
\text { escolarización de estos niños. (C5-N4) }\end{array}$ \\
\hline Nivel 5 & $\begin{array}{l}\text { Incluye información nueva para dar respuesta a la cuestión planteada - cómo se diagnosticaría, qué } \\
\text { actuaciones realizaría cada uno de los implicados en su escolarización, etc.-. Valora distintas } \\
\text { alternativas de escolarización argumentando los pros y los contras de cada una. Expone conclusiones } \\
\text { muy arriesgadas y coherentes. Utiliza conceptos muy elaborados. (C5-N5) }\end{array}$ \\
\hline
\end{tabular}

\subsection{Análisis de los datos}

La codificación y el tratamiento informático de las unidades de información extraídas se ha realizado con el software ATLAS.ti v8. Asimismo, se ha utilizado el paquete estadístico SPSS/PC v24, con el fin de ofrecer una vertiente cuantitativa de los datos obtenidos. Se ha realizado un análisis de conglomerados (clusters) jerárquico con el método de vinculación media entre grupos y distancia euclidea al cuadrado a partir de la agrupación de los estudiantes en función de comportamientos análogos y en relación a las 13 variables sobre las que se había realizado el análisis. Como prueba de homogeneidad de las varianzas se ha utilizado la prueba de Levene y, posteriormente, para conocer la existencia de diferencias estadísticamente significativas el ANOVA de un factor y/o la prueba de Welch.

\section{Resultados}

Se presenta, en primer lugar, la descripción de los niveles de aprendizaje que presentan los estudiantes en relación con las diversas categorías que se han estudiado. En segundo lugar, se establecen perfiles del alumnado en función de los aprendizajes valorados. Y en tercer lugar, se presenta información sobre la influencia tanto del género y como de la participación en cursos piloto de adaptación al EEES sobre el aprendizaje de los estudiantes.

\subsection{Análisis descriptivo}

Se ha realizado un análisis basado en estadísticos descriptivos que pretendía identificar el nivel de aprendizaje (N1 a N5) de los estudiantes en relación a diversas temáticas propias de la formación del pedagogo ( $\mathrm{C} 1$ a $\mathrm{C} 13)$. La tabla 3 recoge las frecuencias obtenidas.

\section{Tabla 3}

Descriptivos sobre las categorías estudiadas 


\begin{tabular}{|c|c|c|c|}
\hline & Media & $\begin{array}{c}\text { Desviación } \\
\text { típica }\end{array}$ & $\mathbf{N}$ \\
\hline C1. Autores de referencia & 2,70 & 1,02 & 47 \\
\hline C2. Currículum & 2,72 &, 80 & 47 \\
\hline C3. Legislación educativa & 2,72 & ,97 & 47 \\
\hline C4. Programas de análisis de datos & 2,62 & 68 & 47 \\
\hline C5. Características Síndrome de Down & 3,28 & ,88 & 47 \\
\hline C6. Modelos de orientación educativa & 2,85 & ,83 & 47 \\
\hline C7. Proyecto de intervención socioeducativa & 3,17 & 60 & 47 \\
\hline C8. Definición y tipos de aprendizaje & 3,00 &, 55 & 47 \\
\hline C9. Sistemas educativos europeos & 2,47 & 1,16 & 47 \\
\hline C10. Educación en valores & 3,06 & 85 & 47 \\
\hline C11. Detección de un alumno con altas capacidades & 3,15 & ,834 & 47 \\
\hline C12. Importancia e implicaciones de la evaluación en educación & 3,23 & ,729 & 47 \\
\hline C13. Formación continua del profesorado & 3,36 & ,764 & 47 \\
\hline
\end{tabular}

Las respuestas de los estudiantes a las cuestiones planteadas $(\mathrm{C} 1$ a $\mathrm{C} 13)$ revelan un tipo de conocimiento más próximo al superficial, codificado, concretamente, entre los niveles uniestructural y multiestructural, con valores medios que oscilan entre 2,47 y 3,68 puntos. Estas puntuaciones describen a un alumnado que tiene dificultades para conceptualizar términos que debe saber manejar (C2. Currículum, C6. Modelos de orientación educativa, C8. Definición y tipos de aprendizaje, C10. Educación en valores). Ofrecen respuestas confusas y poco elaboradas para responder a cuestiones tan básicas como la definición de currículum o la definición de aprendizaje. Estas respuestas, asimismo, no integran una estructura conceptual más amplia que dé muestras de comprensión de dicho contenido:

Por currículum entiendo un artículo en el que aparece un conjunto de aspectos organizativos y metodológicos que una persona posee. Además, éste también incluye las habilidades, destrezas, conocimientos que una persona ha adquirido durante su desarrollo personal, escolar y profesional [P18: Sujeto17.txt - 18:1 (43:45)].

Esta ausencia de una estructura conceptual básica implica que, cuando se les pide que profundicen sobre una temática concreta (C4. Programas de análisis de datos, C5. Características Síndrome de Down e implicaciones educativas, C7. Proyecto de intervención socioeducativa), basen sus explicaciones en aspectos relacionados con el conocimiento cotidiano que ya poseen. De esta manera, aunque son capaces de proporcionar respuestas, no recogen, o lo hacen vagamente, conocimiento experto aprendido en las materias de la titulación:

Las características de los niños con síndrome de Down es que son simpáticos, tienen problemas de visión la mayoría, son muy inquietos. Respecto a su escolarización pueden estar en un colegio ordinario escolarizado, pero con apoyos en aquellas asignaturas que más puedan costarle [P33: Sujeto32.txt - 33:5 (66:68)].

Se observan también dificultades, incluso más pronunciadas, para manifestar la propia perspectiva o posicionamiento respecto a un tópico o temática (C1. Autores de referencia, C3. Legislación educativa, C9. Sistemas educativos europeos). Al respecto, las respuestas de los estudiantes se caracterizan por un desconocimiento sobre la temática que no les permite poseer un criterio formado al respecto, adoleciendo de explicaciones complejas que les permitan tener perspectiva propia o posicionamientos:

Sé muchos, como por ejemplo, Pestalozzi, Rousseau, Montessori, Giner de los Ríos, etc. Pero no sé explicar nada que me hayan portado. Sabría decirte más de Rousseau pero por la ilustración, por la historia [P42: Sujeto41.txt - 42:2 (30:32)].

Por último, las respuestas de los estudiantes en los tres casos prácticos planteados (C11.Detección de un alumno con altas capacidades, C12. Importancia e implicaciones de la evaluación en educación, C13. Formación continua del profesorado), aunque con puntuaciones ligeramente más elevadas, también apuntan a un nivel multiestructural del aprendizaje. Una muestra de ello se muestra en la siguiente respuesta: 
1. ¿Cómo determinarías si se trata de un alumno con altas habilidades? Lo determinaría pasándole ciertas pruebas diagnósticas. 2. ¿Utilizarías alguna prueba o test específico? ¿Cuál o cuáles serían? ¿Dónde los localizarías? El WISC y el TORRANT. Con el WISC medimos su capacidad cognitiva y con el Torrant medimos su creatividad. Las pruebas las pediría para que me las prestaste el equipo de orientación y en su defecto al profesor de diagnóstico. 3. ¿Qué información incluirías en el Informe psicopedagógico? ¿Sería necesario hacer un dictamen de escolarización? ¿En qué caso? Datos del alumno (nombre, edad, colegio, domicilio, teléfono,...), historia del desarrollo (nacimiento, desarrollo personal...), historia escolar, motivo de la evaluación, datos de las pruebas obtenidas... 4. ¿Solicitarías ayuda a algún tipo de agente 0 servicio? ¿Cuál? Sí, solicitaría ayuda al EOEPs de altas capacidades de Murcia y a un centro preferente de niños de altas capacidades. 5. Imagina que, tras la evaluación psicopedagógica, determinas que se trata de un alumno superdotado. ¿Qué medidas contemplarías para dar respuesta educativa a las necesidades educativas del alumno? Posibles opciones: adaptar el currículum; adelantar un curso lectivo; cambiarle de centro a uno preferente de altas habilidades.

En dichos supuestos prácticos, más que usar su conocimiento de forma efectiva y creativa, se han limitado a utilizar el conocimiento declarativo y procedimental para dar respuesta a una situación compleja, observándose ciertas limitaciones relacionadas con el análisis de la realidad, la interpretación y la reflexión argumentada de sus respuestas.

\subsection{Análisis de conglomerados}

En análisis del dendograma permite evidenciar la configuración de dos conglomerados bien definidos. La interpretación de cada uno de ellos es la que sigue:

- El primer clúster lo conforman el $85,1 \%$ del total de los estudiantes (sujetos $6,30,14,36,10,47,33,1$, $25,41,22,46,27,42,3,34,40,2,9,4,17,16,20,29,45,35,32,38,39,23,15,28,8,24,7,43,37,12$, 18,13 ) y se caracteriza por presentar niveles de conocimiento más bajos de la puntuación 3,15 (nivel multiestructural) en las variables analizadas.

- El segundo clúster agrupa al 12,8\% del total de los estudiantes (sujetos 19, 31, 11, 5, 21 y 26), quienes evidencian un nivel de conocimiento más elevado (igual o superior a la puntación 3,15 ) respecto a las diversas variables analizadas.

La explicación de los dos conglomerados emergidos da muestra de dos perfiles diferenciados de estudiantes, aquellos que muestran un aprendizaje meramente superficial y aquellos que manifiestan un aprendizaje próximo al profundo a cerca de los tópicos planteados. Se aprecia que la división entre los dos conglomerados se sitúa en el sujeto 44.

Influencia del género sobre el aprendizaje de los estudiantes

Se presentan los resultados en relación con el género de los participantes. En la siguiente tabla figuran las medias y desviaciones típicas halladas.

Tabla 4

Estadísticos según género

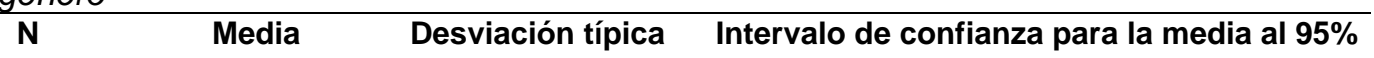

\begin{tabular}{lccccc}
\cline { 4 - 5 } & & & Límite inferior & Límite superior \\
\hline Mujeres & 43 & 35,26 & 4,89 & 33,75 & 36,76 \\
Hombres & 4 & 42,50 & 8,23 & 29,41 & 55,59 \\
Total & 47 & 35,87 & 5,52 & 34,25 & 37,49 \\
\hline
\end{tabular}

Como se puede apreciar, los datos revelan un porcentaje medio más elevado en favor de los hombres. A fin de comprobar si las diferencias encontradas en las puntuaciones medias obtenidas por alumnos y alumnas son estadísticamente significativas, se aplica la prueba Welch, ya que previamente la prueba de Levene indica que las varianzas son heterogéneas: $p=0,97$, es decir, no se verifica el supuesto de heterocedasticidad. Este 
estadístico permite comprobar que no existen diferencias significativas entre alumnos y alumnas en cuanto a los niveles de aprendizaje desarrollados.

Influencia de la participación en cursos piloto de adaptación al EEES sobre el aprendizaje de los estudiantes

Se presentan los valores medios de las puntuaciones obtenidas por los estudiantes según su participación o no en los cursos piloto de adaptación al grado. Los datos arrojados muestran que, en casi todas las variables a excepción de autores de referencia y sistemas educativos europeos, las puntuaciones más elevadas las obtienen los estudiantes que no han participado en ningún curso piloto en detrimento de aquellos que sí lo han hecho, alcanzando -los primeros- puntuaciones superiores a 3.

Tabla 5

Estadísticos descriptivos según participación en curso piloto

\begin{tabular}{|c|c|c|c|c|}
\hline & & $\mathbf{N}$ & Media & $\begin{array}{l}\text { Desviación } \\
\text { típica }\end{array}$ \\
\hline & Sí & 42 & 2,74 & 1,01 \\
\hline \multirow[t]{2}{*}{ Autores de referencia } & No & 5 & 2,40 & 1,14 \\
\hline & Total & 47 & 2,70 & 1,02 \\
\hline & Sí & 42 & 2,64 &, 73 \\
\hline \multirow[t]{3}{*}{ Currículum } & No & 5 & 3,40 & 1,14 \\
\hline & Total & 47 & 2,72 &, 80 \\
\hline & Sí & 42 & 2,60 & ,86 \\
\hline \multirow[t]{3}{*}{ Legislación educativa } & No & 5 & 3,80 & 1,30 \\
\hline & Total & 47 & 2,72 &, 97 \\
\hline & Sí & 42 & 2,57 & ,63 \\
\hline \multirow[t]{3}{*}{ Programas de análisis de datos } & No & 5 & 3,00 & 1,00 \\
\hline & Total & 47 & 2,62 &, 68 \\
\hline & Sí & 42 & 3,21 & 87 \\
\hline \multirow[t]{3}{*}{ Características del síndrome de Down } & No & 5 & 3,80 & ,84 \\
\hline & Total & 47 & 3,28 & ,88 \\
\hline & Sí & 42 & 2,79 &, 72 \\
\hline \multirow[t]{3}{*}{ Modelos de orientación educativa } & No & 5 & 3,40 & 1,52 \\
\hline & Total & 47 & 2,85 &, 83 \\
\hline & Sí & 42 & 3,17 &, 58 \\
\hline \multirow[t]{3}{*}{ Proyecto de intervención socioeducativa } & No & 5 & 3,20 & ,84 \\
\hline & Total & 47 & 3,17 &, 60 \\
\hline & Sí & 42 & 2,93 &, 51 \\
\hline \multirow[t]{3}{*}{ Definición y tipos de aprendizaje } & No & 5 & 3,60 &, 55 \\
\hline & Total & 47 & 3,00 &, 55 \\
\hline & Sí & 42 & 2,50 & 1,15 \\
\hline \multirow[t]{3}{*}{ Sistemas educativos europeos } & No & 5 & 2,20 & 1,30 \\
\hline & Total & 47 & 2,47 & 1,16 \\
\hline & Sí & 42 & 3,02 & ,72 \\
\hline \multirow[t]{3}{*}{ Educación en valores } & No & 5 & 3,40 & 1,67 \\
\hline & Total & 47 & 3,06 &, 85 \\
\hline & Sí & 42 & 3,12 & 80 \\
\hline \multirow[t]{2}{*}{ Detección de un alumno con altas capacidades } & No & 5 & 3,40 & 1,14 \\
\hline & Total & 47 & 3,15 &, 83 \\
\hline \multirow{3}{*}{$\begin{array}{l}\text { Importancia e implicaciones de la evaluación } \\
\text { educación }\end{array}$} & Sí & 42 & 3,21 & ,68 \\
\hline & en $_{\text {No }}$ & 5 & 3,40 & 1,14 \\
\hline & Total & 47 & 3,23 &, 73 \\
\hline \multirow{3}{*}{ Formación continua del profesorado } & Sí & 42 & 3,26 &, 70 \\
\hline & No & 5 & 4,20 & ,84 \\
\hline & Total & 47 & 3,36 & ,76 \\
\hline
\end{tabular}

Para comprobar si las diferencias encontradas son estadísticamente significativas se aplica, de nuevo, la prueba de Levene, constatando que existen varianzas homogéneas para las variables Modelos de orientación educativa $(p=0,00)$ y Educación en valores $(p=0,00)$; y varianzas heterogéneas para las demás variables 
$(p>5)$. Se realiza el ANOVA de un factor, con objeto de analizar la existencia de diferencias significativas en relación a dichas variables. Los resultados obtenidos permiten comprobar que en ninguna de dichas variables existen diferencias significativas entre el alumnado según su participación en experiencias de plan piloto.

Del mismo modo, a fin de conocer la existencia de diferencias significativas del resto de variables se aplica la prueba de Welch, comprobando, en este caso, la existencia de diferencias estadísticamente significativas en la variable relativa a Definición y tipos de aprendizaje, con un valor $p<0,05$. Dichas diferencias se observan a favor de los estudiantes que no habían participado en ningún a experiencia piloto.

\section{Discusión y conclusiones}

El estudio expuesto muestra la existencia de dos grupos o tipologías de estudiantes en relación a la calidad de las respuestas dadas a diversas cuestiones y tareas auténticas relacionadas con su formación académica. Por un lado, se identifica un grupo amplio de estudiantes -la mayoría de los participantes de este estudio-, caracterizado por proporcionar respuestas poco elaboradas, referidas a un conocimiento cotidiano y desconectadas de la realidad profesional, presentando, asimismo, dificultades para abordar explicaciones detalladas y complejas y para realizar valoraciones e interpretaciones sobre cuestiones curriculares propias de su formación. Por otro lado, se identifica un grupo de estudiantes menos numeroso (que representa una minoría) caracterizado, en este caso, por presentar, mínimamente, respuestas de mayor calidad, entendiendo éstas como respuestas que integran una mayor comprensión de los contenidos y explicaciones un poco más detalladas sobre las cuestiones planteadas. Estos resultados coinciden con investigaciones previas realizadas a lo largo de los últimos 30 años a nivel internacional (McDermott, 1984; West, 1988; Ramsden, 2003; Grácio, Chaleta y Rosário, 2005) y permiten dilucidar que persisten las limitaciones de estos estudiantes cuando acaban su formación universitaria para ir más allá de la reproducción mecánica de conocimientos y para adaptarse a nuevos escenarios e imprevisibles situaciones educativas.

Otro de los objetivos ha sido determinar la existencia de diferencias estadísticamente significativas en cuanto al nivel de conocimiento en relación al sexo de los estudiantes. Al respecto, se corrobora que el nivel de conocimiento del alumnado en relación a los contenidos de formación sobre los que se les ha preguntado es similar a nivel de género. En este sentido, se puede concluir que no se hallan diferencias estadísticamente significativas entre el conocimiento desarrollado por los alumnos y las alumnas de la titulación de Pedagogía. Es importante destacar, además, que tampoco se detectan diferencias estadísticamente significativas según la participación o no de los estudiantes en cursos piloto donde se pusieron en marcha metodologías más innovadoras y participativas. En coherencia con esta débil relación encontrada, la investigación realizada por González y Cutanda (2017) refleja la dificultad de conectar el impacto de las prácticas docentes más o menos innovadoras con los aprendizajes que se están posibilitando en los estudiantes, aunque en ese caso la investigación fue realizada en Educación Secundaria. Otros hallazgos a nivel internacional, como el realizado por Hightower et al. (2011) también ha puesto de manifiesto las dificultades que suponen conectar el grado de incidencia de prácticas docentes innovadoras y participativas en las aulas (tras un proceso de desarrollo profesional docente) y los aprendizajes de los estudiantes. En este sentido, las asociaciones que se establecen entre prácticas de enseñanza y aprendizajes de los estudiantes han de tomarse con cierta cautela por entrar en juego otros factores contextuales que también pueden determinar el aprendizaje de los estudiantes. Así pues, parece coherente que los resultados de este estudio muestren que, en principio, los estudiantes que se han formado a través del modelo de enseñanza actual no han evidenciado aprendizajes de mayor calidad. En esta línea, cabe destacar que, posiblemente, tampoco se habían asentado prácticas docentes alternativas, bien estructuradas y engranadas, que implicaran activamente y comprometidamente a los estudiantes en su aprendizaje. O es posible, asimismo, que esa filosofía de cambio hacia una enseñanza centrada en el aprendizaje estuviese esté en proceso de ser asumida y compartida por toda la comunidad educativa, tanto por el profesorado como por el alumnado. En esta línea se podría plantear como línea de trabajo complementaria, volver a realizar esta investigación una vez se hayan salvado los obstáculos que se presentan cuando se produce un cambio de modelo educativo de esta envergadura, pues, aun estando ratificado que la introducción de métodos centrados en el aprendizaje potencian el aprendizaje profundo de los estudiantes (Gargallo, Sahuquillo, Verde y Almerich, 2018), es necesario que dichos cambios se asienten y, para ello, se necesita tiempo. 
A pesar de ello, estos resultados podrían ser relacionados con otros estudios, citados al inicio de este artículo, que aluden tanto a variables contextuales como personales para explicar los resultados de aprendizaje de los estudiantes. Entre otros factores, dichos estudios destacan las estrategias y enfoques de aprendizaje de los estudiantes como variables que influyen directamente en la calidad de sus aprendizajes, poniendo de manifiesto que los estudiantes con resultados de aprendizaje más elevados se decantan más por enfoques de aprendizaje profundo y estudiantes con resultados de aprendizaje más bajos utilizan enfoques más superficiales para aprender, y manifestando, a su vez, que los enfoques profundos llevarían a aprendizajes comprensivos y más duraderos en el tiempo mientras los enfoques superficiales supondrían aprendizajes de menor duración. Estas apreciaciones permiten justificar, con discutibles matizaciones, que los estudiantes podrían haber utilizado enfoques superficiales para aprender e incluso para abordar las tareas y cuestiones planteadas en este estudio. También se podría inferir que estos estudiantes poseen metas de logro, expectativas y motivaciones inadecuadas hacia las tareas e, incluso, hacia la profesión; que podría provenir del propio alumnado pero también de la utilidad y el valor de la educación que se transmite desde la labor docente (Sancho-Álvarez, Jornet y González-Such, 2016).

Estos datos, por tanto, dilucidan la necesidad de la comunidad universitaria de continuar esforzándose por alcanzar aprendizajes de calidad: promoviendo metas de alto nivel cognitivo, suscitando expectativas en los estudiantes que les permitan aprender con mayor motivación, favoreciendo el pensamiento crítico y generando una actitud positiva hacia la educación y hacia la propia formación. Adoptar un enfoque profundo depende también de la tarea de aprendizaje que se proponga (López-Alonso, López-Aguado, FernándezMartínez, Liébana-Presa y Gutiérrez-Provecho, 2016); en este sentido, es imprescindible el compromiso de los docentes y de toda la comunidad universitaria para reorientar sus prácticas educativas y favorecer en los estudiantes estrategias y enfoques de aprendizaje que los lleven a la comprensión y a la significación personal.

\section{Referencias}

Arias, O. y Fidalgo, R. (2013). Innovación educativa en la Educación Superior. Madrid, España: Editorial Académica Española.

Biggs, J. (2005). Calidad en el aprendizaje universitario. Madrid, España: Narcea.

Biggs, J. y Collis, K. (1982). Evaluating the Quality of Learning: the SOLO taxonomy. Nueva York, NY: Academy Press. Biggs, J. y Tang, C. (2011). Theaching for Quality Learning at University. What the student does (4 $4^{a}$ Ed.). Nueva York, NY: Open University Press.

Bisquerra, R. (Coord.) (2012). Metodología de la Investigación Educativa (3 $3^{a}$ Ed.). Madrid, España: La Muralla S.A. Buendía, L. y Olmedo, E. M. (2003). Estudio transcultural de los enfoques de aprendizaje en educación superior. Revista de Investigación Educativa, 21(2), 511-524. Recuperado de https://revistas.um.es/rie/article/view/99261/94861

Delors, J. (1996). La educación encierra un tesoro. Madrid, España: Santillana.

Duque, M., Celis, J., Díaz, B. y Gómez, M. (2014). Diez pilares para un programa de desarrollo profesional docente centrado en el aprendizaje de los estudiantes. Revista Colombiana de Educación, 67, 107-124. Recuperado de http://www.scielo.org.co/pdf/rcde/n67/n67a06.pdf

Entwistle, N. (1986). Approaches to Learning in Higher Education: Effects of Motivation and Perceptions of the Learning Environment. Annual Meeting of the American Educational Research Association, San Francisco.

Esquivel, J., Rodríguez, M. C. y Padilla, V. M. (2009). Enfoques hacia el aprendizaje, motivos y estrategias de estudiantes de las carreras de enfermería, ingeniería y organización deportiva. Revista de Pedagogía, 30(87), 309-331. Recuperado de http://www.redalyc.org/articulo.0a?id=65914495005

Forés, A. y Subias, E. (Eds.) (2017). Pedagogías emergentes. 14 preguntas para el debate. Barcelona, España: Octaedro.

Gabay, C. (2015). Special Forum on the Millennium Development Goals: Introduction. Globalizations, 12(4), 576-580. DOI: https://doi.org/10.1080/14747731.2015.1033173

Gadelrab, H. F. (2011). Approaches and Study Skills Inventory for Students (ASSIST) in Egypt: A confirmatory factor analysis approach. Electronic Journal of Research in Educational Psychology, 9(3), 1197-1218. Recuperado de http://ojs. ual.es/ojs/index.php/EJREP/article/viewFile/1501/1804

García-Córdoba, F. (2004). Recomendaciones metodológicas para el diseño de un cuestionario. México: Limusa. Gargallo, B., Garfella, P.R., Sahuquillo, P.M., Verde, I., Jiménez, M.A. (2015). Métodos centrados en el aprendizaje, estrategias y enfoques de aprendizaje en estudiantes universitarios. Revista de Educación, 370, 229-254. DOI: https://10.4438/1988-592X-RE-2015-370-304

Gargallo, B., Sahuquillo M., Piedad, M., Verde, I. y Almerich, G. (2018). ¿Qué ocurre cuando los profesores utilizan métodos centrados en el aprendizaje? Efectos en los enfoques de aprendizaje, en las capacidades del alumno y en su percepción del entorno de aprendizaje. Revista de Educación, 382, 163-197. DOI: 10.4438/1988-592X-RE-2018-382396 
Gargallo, B., Suárez, J., García, E., Pérez, C. y Sahuquillo, P.M. (2012). Enfoques de aprendizaje en estudiantes universitarios excelentes y en estudiantes medios. Revista Española de Pedagogía, 252, 185-200. Recuperado de https://revistadepedagogia.org/wp-content/uploads/2012/05/252-01.pdf

George, A. L. y Bennett, A. (2005). Case studies and theory development in the social sciences. Cambridge, MA: MIT Press.

Gil-Galván, R. (2018). El uso del aprendizaje basado en problemas en la enseñanza universitaria. Análisis de las competencias adquiridas y su impacto. Revista Mexicana de Investigación Educativa, 23(76), 73-93. Recuperado de https://www.comie.org.mx/v1/revista/visualizador.php?articulo=ART76003\&criterio=http://www.comie.org.mx/documento s/rmie/v23/n076/pdf/76003.pdf

González, M. T. y Cutanda, M. T. (2017). Formación continuada del profesorado, mejora de la enseñanza y de los aprendizajes del alumnado. Revista Mexicana de Investigación Educativa, 22(75), 1095-1116. Recuperado de https://www.comie.org.mx/v1/revista/visualizador.php?articulo=ART75004\&criterio=http://www.comie.org. $\mathrm{mx} / \mathrm{documento}$ s/rmie/v22/n075/pdf/75004.pdf

Grácio, L., Chaleta, E. y Rosário, P. (2005). Emergência de novas concepções de aprendizagem e ensino e suas implicações educativas. Trabalho apresentado em VIII Congresso Galaico-Português de Psicopedagogia. VIII Congresso Galaico-Português de Psicopedagogia, Braga.

Guzmán, J. C. (2011). La calidad de la enseñanza en educación superior. ¿Qué es una buena enseñanza en este nivel educativo? Perfiles Educativos, 3 (número especial), 129-241. Recuperado de http://www.scielo.org.mx/scielo.php?script=sci arttext\&pid=S0185-26982011000500012

Hightower, A. M., Delgado, R. C., Lloyd, S. C., Wittgenstein, R., Sellers, K. y Swanson, C. B. (2011). Improving student learning by supporting quality teaching: key issues, effective strategies. Bethesda: Editorial Projects in Education, Inc.

Recuperado de https://www.edweek.org/media/eperc qualityteaching_12.11.pdf

López-Alonso, A. I., López-Aguado, M., Fernández-Martínez, M. E., Liébana-Presa, C. y Gutiérrez-Provecho, L. (2016). Los enfoques de aprendizaje, el engagement, el ocio y el rendimiento anterior. Propuesta de un modelo. Bordón, 68(4), 67-88. DOI: https://10.13042/Bordon.2016.40940

Maquilón, J. y Hernández-Pina, F. (2011). Influencia de la motivación en el rendimiento académico de los estudiantes de formación profesional. Revista Electrónica Interuniversitaria de Formación del Profesorado, 14(1), 81-100. Recuperado de http://www.redalyc.org/articulo.oa?id=217017192007

Martínez, F. M. y Carmona, G. (2009). Aproximación al concepto de "competencias emprendedoras": valor social e implicaciones educativas. REICE. Revista Iberoamericana sobre Calidad, Eficacia y Cambio en Educación, 7(3), 82-98. Recuperado de https://revistas.uam.es/index.php/reice/article/view/5403/5842

Marton, F. y Säljö, R. (1976a). On qualitative differences in learning-I: Outcome and process. British Journal of Educational Psychology, 46, 4-11.

Marton, F. y Säljö, R. (1976b). On qualitative differences in learning-II: Outcome as a function of the learner`s conception of the task. British Journal of Educational Psychology, 46, 115-127.

McDermott, L. C. (1984). Research on conceptual understanding in mechanics. Physics Today, 37, $24-32$.

Merriam, S. B. y Tisdell, E. J. (2016). Qualitative research: a guide to design and implementation (4th edition). San Francisco, CA: Jossey-Bass.

Monereo, C. y Pozo, J.I. (2003). La universidad ante la nueva cultura educativa. Enseñar y aprender para la autonomía. Madrid, España: Síntesis.

Navarro, E. (2012). La enseñanza de la Historia de España y el desarrollo de las competencias ciudadanas. El conocimiento del alumnado al finalizar el Bachillerato. (Tesis doctoral, no publicada). Universidad de Murcia, Murcia. Olmedo, E. (2013). Enfoques de aprendizaje de los estudiantes y metodología docente: Evolución hacia el nuevo sistema de formación e interacción propuesta en el EEES. Revista de Investigación Educativa, 31(2), 411-429. DOI: http://dx.doi.org/10.6018/rie.31.2.133501

Pérez-Gómez, A. (Ed.) (2009). Aprender cómo aprender. Autonomía y responsabilidad: el aprendizaje de los estudiantes. Madrid, España: Akal.

Pérez-Serrano, G. (1994). Investigación cualitativa. Retos, interrogantes y métodos. Madrid, España: La Muralla. Pozo, J.I. y Monereo, C. (2009). Introducción: La nueva cultura del aprendizaje universitario o por qué cambian nuestras formas de enseñar y aprender. En J.I. Pozo y M.P. Pérez (coords.), Psicología del aprendizaje universitario: La formación en competencias (pp.9-28). Madrid, España: Ediciones Morata.

Ramsden, P. (2003). Learning to Teach in Higher Education (2 $2^{\mathrm{a}}$ Ed.). Londres: Routledge.

Riesco, M. (2008). El enfoque por competencias en el EEES y sus implicaciones en la enseñanza y el aprendizaje. Tendencias Pedagógicas, 13, 79-105.

Ritchhart, R., Church, M. y Morrison, K. (2014). Hacer visible el pensamiento. Cómo promover el compromiso, la comprensión y la autonomía de los estudiantes. Buenos Aires: Paidós.

Rosário, P. y Almeida, L. (2005). Leituras constructivistes da aprendizagem. En G. L. miranda, y S. Badia (Org.), Psicologia da Educaçâo. Temas de desenvolmiento, aprendizagem e ensino (pp. 141-146). Lisboa, Portugal: Relógio D’Água.

Roys, J. y Pérez, A. (2018). Estrategias de aprendizaje significativo en estudiantes de educación superior y su asociación con logros académicos. Revista Electrónica de Investigación y Docencia (REID), 19, 145-166. DOI: https://dx.doi.org/10.17561/reid.v0i19.3570 
Salim, R. (2006). Motivaciones, enfoques y estrategias de aprendizaje en estudiantes de Bioquímica de una universidad pública argentina. Revista Electrónica de Investigación Educativa, 8(1). Recuperado de

https://redie.uabc.mx/redie/article/view/137/236

Sancho-Álvarez, C., Jornet, J. M. y González-Such, J. (2016). El constructo valor social subjetivo de la Educación:

validación cruzada entre profesorado de escuela y universidad. Revista de Investigación Educativa, 34(2), 329-350. DOI: http://dx.doi.org/10.6018/rie.34.2.226131

Sancho-Álvarez, C., Jornet, J. M. y Perales, J. (2014). Valor social subjetivo de la educación: docencia y resultados escolares. Revista Iberoamericana de Evaluación Educativa (RIEE), 7(2), 197-207. Recuperado de http://www.rinace.net/riee/numeros/vol7-num2e/art15.pdf

Stewart, V. (2012). A world-class education: learning from international models of excellence and innovation. The United States of America: Ascd.

Vázquez, J. A. (2015). Nuevos escenarios y tendencias universitarias. Revista de Investigación Educativa, 33(1), $13-26$. DOI: http://dx.doi.org/10.6018/rie.33.1.211501

West, L. H. T. (1988). Implications of recent research for improving secondary school science learning. En P. Ramsden (Ed.), Improving Learning: New Perspectives. Londres: Kogan Page.

Zabalza, M.A. (2011). Metodología docente. REDU-Revista de Docencia Universitaria, 9(3), 75-98.

DOI: https://doi.org/10.4995/redu.2011.6150 